Ensino, Saúde e Ambiente - V9 (3), pp. 84-99, Dez. 2016

\title{
ANÁLISE DOS ARTIGOS DA REVISTA ELETRÔNICA ENSINO, SAÚDE E AMBIENTE QUE VERSEM SOBRE "SAÚDE"
}

\section{ANALYSIS OF ARTICLES OF THE ELECTRONIC JOURNAL EDUCATION, HEALTH AND ENVIRONMENT CONTAINING THE DESCRIPTOR "HEALTH" IN ITS TITLE}

\author{
Sonia Teresinha Flores dos Santos ${ }^{1}$, Julio Cesar Bresolin Marinho ${ }^{2}$, Edward Frederico \\ Castro Pessano \\ ${ }^{1}$ Licenciada em Ciências da Natureza e Especialista em Educação em Ciências \\ E-mail: soniafloressantos@gmail.com \\ ${ }^{2}$ Professor da Universidade Federal do Pampa (UNIPAMPA) - Campus Uruguaiana \\ E-mail: juliomarinho@unipampa.edu.br \\ ${ }^{3}$ Professor da Universidade Federal do Pampa(UNIPAMPA) - Campus Uruguaiana \\ E-mail: edwardpessano@unipampa.edu.br
}

\begin{abstract}
RESUMO
A investigação trata-se de um estudo de natureza qualitativa, Estado da Arte, cujo propósito foi analisar o 'objetivo' dos artigos publicados na Revista Eletrônica "Ensino, Saúde e Ambiente" que continham o descritor "saúde" em seu título. Foram analisados 39 , de um total de 204 artigos, que foram publicados no período de 2008 , quando a revista foi criada, até 2015. Os artigos selecionados foram analisados em duas categorias, a partir de sua abordagem da temática: locais de foco dos estudos e categorização dos objetivos dos artigos. Optamos por essa forma de apresentar os resultados, afim de um melhor entendimento e análise dos dados e de organizar as discussões. A relevância do tema escolhido deu-se pela importância de verificar os objetivos aos quais se propuseram, contribuindo para uma avaliação das produções por este importante periódico, o qual é uma das referências para a área de Educação em Saúde e para o campo do Ensino de Ciências. Como resultado das análises verificou-se a maior frequência de pesquisas efetuadas em escolas, com objetivos predominantes na avaliação de novas estratégias de ensino e aprendizagem.
\end{abstract}

Palavras-chave: Estado da Arte; Educação em Saúde; Saúde; Ensino de Ciências.

\begin{abstract}
This research is a qualitative study, State of Art, which purpose was to analyze the 'purpose' of the articles published in the Electronic Journal "Education, Health and Environment" containing the descriptor "health" in its title. There were analyzed 39 in 204 articles, published from 2008, when the magazine was created, to 2015. The selected articles were analyzed in two categories, from its thematic approach: the location of focus of studies and categorization of the articles' purposes. We opted for this way of presenting the results in order to better understand and analyze data, and organize the discussions. The relevance of the chosen topic was due to the importance of verifying the purposes to which they proposed, contributing to an assessment of production made by this important journal, which is one of the references to Health Education area and to Science Education field. As a result of the analysis, it was found a higher frequency of research conducted in schools, with overriding purposes on the evaluation of new teaching and learning strategies.
\end{abstract}

Key-words: State of Art; Health Education; Health; Science Education. 


\title{
INTRODUÇÃOO
}

A revista eletrônica "Ensino, Saúde e Ambiente" teve seu primeiro número publicado em agosto de 2008, apresenta periodicidade quadrimestral (abril, agosto e dezembro) e possui ênfase no Ensino de Ciências com interface em educação para o ambiente e educação para a saúde. $\mathrm{O}$ foco e escopo da revista residem na:

\begin{abstract}
publicação de artigos científicos resultantes de pesquisas e de relatos de experiências originais sobre temas que envolvem o Ensino de Ciências e suas interfaces com as questões do Ambiente e da Saúde. Desde a sua primeira edição em 2008 mantém na divulgação dos resultados das pesquisas que vêm sendo desenvolvidas na área de Ensino de Ciências, com ênfase na tríade Ensino-Saúde-Ambiente. Tem ainda como compromisso disseminar esta produção à pesquisadores, professores e estudantes de todos os níveis de ensino, bem como ao público interessado em geral (REVISTA ENSINO, SAÚDE E AMBIENTE, 2015).
\end{abstract}

O periódico é classificado pelo sistema Qualis CAPES em oito áreas de classificação: Educação, Ensino, Interdisciplinar, Ciência Política e Relações Internacionais, Engenharia III, Letras/Linguística, Ciências Biológicas II e Química (Qualis 2014). Tal evidência reforça o caráter interdisciplinar da revista. A revista, ainda possui o compromisso de disseminar esta produção a pesquisadores, professores e estudantes de todos os níveis de ensino, bem como ao público interessado em geral. Isso demonstrou a necessidade de investigar e problematizar os artigos que discutem a temática "saúde" publicada na revista aqui estudada.

A proposta desta pesquisa surgiu no decorrer do Curso de Especialização em Educação em Ciências, pela Universidade Federal do Pampa (UNIPAMPA), Campus Uruguaiana - RS, devido à atenção que a revista está disponibilizando aos artigos referentes à Educação em Saúde, área a qual também é incluída nas discussões e nos focos das pesquisas do referido Programa de Pós-Graduação. Desta forma, nossa atenção residiu nos artigos que, foram desenvolvidos e publicados com essa temática e dando ênfase nos seus objetivos, a fim de compreender a sua contribuição para o campo da Educação em Saúde.

A Organização Mundial de Saúde (OMS) tem como conceito de saúde "o estado do mais completo bem-estar físico, mental, social e espiritual, e não apenas como ausência de doenças e fraquezas" (WEINECK, 2003, p. 20). Ao conscientizar as pessoas para o direito à saúde, sensibilizá-los para a busca permanente da compreensão e capacitar para a utilização de medidas práticas de promoção, proteção e recuperação da saúde ao seu alcance, somente assim é que a Educação para a Saúde cumprirá seus objetivos. Melo (1987 apud SCHALL e STRUCHINER, 1999, p. 3) nos diz que: 
é necessário pensar a Educação e a Saúde não mais como uma educação sanitarizada (educação sanitária) ou localizada no interior da saúde (educação em saúde) ou ainda educação para a saúde (como se a saúde pudesse ser um estado que se atingisse depois de educado!). É preciso recuperar a dimensão da Educação e da Saúde/doença e estabelecer as articulações entre esses dois campos e os movimentos (organizados) sociais. E mais - como práticas sociais articuladas com as necessidades e possibilidades das classes populares na formulação de políticas sociais e das formas de organização social que lhes interessam.

Cabe destacar que ocorreu uma mudança de nomenclatura, por volta da década de 70, passa de educação sanitarizada para ser Educação para Saúde, (LEVY et al, 1997). Assim, mais que uma mudança terminológica, começava a partir de então, um novo conceito na promoção da saúde.

Conforme Vasconcelos (1998) a Educação em Saúde surge como um instrumento de construção da participação popular nos serviços de saúde e, ao mesmo tempo, de aprofundamento da intervenção da ciência na vida cotidiano das famílias e sociedades. Silva e Juliani (2010) manifestam que a Educação em Saúde é um processo de trocas de saberes e experiências entre a população como um todo, incluindo seus usuários e profissionais, sendo que cada pessoa é valorizada como um aprendiz e um educador.

Entende-se que o estudo sobre o tema "saúde" se faz pertinente, pois, verifica-se que a área da Educação em Saúde, apresenta crescimento em suas publicações científicas, fator que aponta a necessidade de sua avaliação, bem como a verificação da contribuição desta produção para o campo da Educação. Neste sentido, esse trabalho se propôs a analisar os artigos da Revista Eletrônica Ensino, Saúde e Ambiente, o qual serve de referência para variados trabalhos de pesquisa dentro dos processos de ensino e aprendizagem voltados à promoção da saúde. Além disso, analisar as produções da revista, em relação à saúde, pode apontar o direcionamento das investigações e qual a sua contribuição real para a educação e Ensino de Ciências. Acreditamos também que o trabalho poderá contribuir, evidenciando possíveis lacunas existentes, bem como apontar a necessidade de outros estudos que podem ser explorados a partir desta temática, como, por exemplo, a avaliação das estratégias de ensino para a promoção da saúde.

Portanto, a presente investigação tem como finalidade avaliar os trabalhos já publicados na Revista Eletrônica Ensino, Saúde e Ambiente, verificando os objetivos aos quais se propõem os artigos, contribuindo para uma avaliação das produções deste periódico desde sua criação em 2008 até o ano de 2015. 


\title{
EDUCAÇÃO EM SAÚDE: O QUE A LITERATURA NOS MOSTRA?
}

Fonseca (1994, p. 27) nos apresenta uma visão de Educação em Saúde bem comportamentalista e restrita, como um conceito que se emprega como sinônimo de ensino de saúde, sendo compreendido como a "transmissão de conhecimentos e informações, mas principalmente no desenvolvimento de hábitos, atitudes, habilidades e comportamentos que ajudem na promoção, proteção, conservação, recuperação e reabilitação da saúde”. Santos (1999) já nos mostra a importância da relevância do tema, dirigindo-se a três beneficiários: a sociedade, a ciência e a escola. Schall e Struchiner (1999, p. 4) nos apresentam uma compreensão de Educação em Saúde que se entrelaça com o conceito da promoção da saúde:

\begin{abstract}
Ao conceito de educação em saúde se sobrepõe o conceito de promoção da saúde, como uma definição mais ampla de um processo que abrange a participação de toda a população no contexto de sua vida cotidiana e não apenas das pessoas sob risco de adoecer. Essa noção está baseada em um conceito de saúde ampliado, considerado como um estado positivo e dinâmico de busca de bem-estar, que integra os aspectos físico e mental (ausência de doença), ambiental (ajustamento ao ambiente), pessoal/emocional (auto-realização pessoal e afetiva) e sócio-ecológico (comprometimento com a igualdade social e com a preservação da natureza).
\end{abstract}

Conforme as autoras discorrem acima, a Educação em Saúde, não se sustenta sozinha precisa de elos entre diversos segmentos da sociedade, para que se consiga chegar o mais perto possível da promoção da saúde da sociedade.

As políticas de saúde reconhecem o espaço escolar como espaço privilegiado para práticas promotoras da saúde, preventivas e de educação para saúde. O Programa "Mais Saúde: Direito de Todos", lançado pelo Ministério da Saúde é um exemplo disso (BRASIL, 2008b). O Programa Saúde na Escola (PSE), instituído por Decreto Presidencial $n^{\circ}$ 6.286, de 5 de dezembro de 2007 (BRASIL, 2007), resulta do trabalho integrado entre o Ministério da Saúde e o Ministério da Educação, na perspectiva de ampliar as ações específicas de saúde aos alunos da rede pública de ensino: Ensino Fundamental, Ensino Médio, Rede Federal de Educação Profissional e Tecnológica, Educação de Jovens e Adultos (BRASIL, 2008a). Por conseguinte o Ministério da Saúde pressupõem que as práticas pedagógicas e sociais devem ser compartilhadas pelos profissionais da área em conjunto com os profissionais da educação.

O Ministério da Saúde (1993, p. 13) entende Educação em Saúde como:

um conjunto de práticas pedagógicas e sociais, de conteúdo técnico, político e científico, que no âmbito das práticas de atenção à saúde deve ser vivenciada e compartilhada pelos trabalhadores da área, pelos setores organizados da população e consumidores de bens e serviços de saúde. 
Assim, concordamos com Mohr (2002) que sustenta que a escola tem o importante papel de desenvolver conteúdos de forma tal que os conhecimentos necessários para análises de situações relacionadas à saúde estejam disponíveis para o indivíduo. Segundo a autora:

\begin{abstract}
A ES [Educação em Saúde] deve ser encarada pela escola como o objetivo geral de desenvolvimento da capacidade humana. Ela não deve ser considerada uma atividade-meio através da qual, em curto prazo, se atingirão determinadas atitudes, hábitos e comportamentos. Tampouco a escola pode ser considerar que tem controle e jurisdição sobre estes elementos e, portanto, poderia obedece-los univocamente e avaliar se no fim de dado período letivo, os alunos o alcançaram ou não. A ES na escola deve ser considerada, na realidade, um objetivo ao qual se chega de forma indireta (MOHR, 2002, p. 242).
\end{abstract}

Ainda uma percepção proposta por Mohr (2002, p. 38), onde Educação em Saúde é compreendida como o conjunto de "atividades realizadas como parte do currículo escolar, que tenham uma intenção pedagógica definida, relacionada ao ensinoaprendizagem de algum assunto ou tema relacionado com a saúde individual ou coletiva". Nesse sentido cabe ao professor estar apto a conduzir práticas de Educação em Saúde e colaborar com a aprendizagem correlata.

As Diretrizes da Educação para a Saúde (BRASIL, 1980, p. 370) definem Educação em Saúde como "uma atividade planejada que objetiva criar condições para produzir as mudanças de comportamento desejadas em relação à saúde". Assim, esse trabalho subentende aqui, que a Educação em Saúde é uma dimensão das práticas de saúde que envolve docentes, profissionais da saúde e poder público e deverá ser alcançada mediante interações de ações dos sujeitos.

\title{
METODOLOGIA
}

O trabalho foi desenvolvido durante o segundo semestre de 2015 e primeiro semestre de 2016. Sendo uma pesquisa de caráter qualitativo, baseada na investigação teórica dos artigos científicos, publicados na revista eletrônica "Ensino, Saúde e Ambiente", que continham o descritor "saúde" em seus títulos, buscando analisar os objetivos de cada produção, durante o período de 2008 a 2015, o qual caracteriza desde a origem do periódico até seu último número no ano de 2015.

A pesquisa configura-se como qualitativa do tipo Estado da Arte ou Estado do Conhecimento, pois essa metodologia possibilita a organização, análise e revisão de 
artigos já publicados. De acordo com Santos et al. (2006, p. 16), "a geração de conhecimento deve iniciar com a pesquisa do que já foi previamente publicado a respeito do tema estudado". Sendo assim o Estado da Arte ou do Conhecimento permite ao pesquisador analisar produções do estado atual, a partir das revisões bibliográficas para possibilitar a fundamentação do trabalho científico.

Soares (1989) salienta que esses estudos, do Estado da Arte, são necessários no processo de evolução da ciência, a fim de que se ordene periodicamente o conjunto de informações e resultados já obtidos. Um Estado da Arte pode constituir-se em levantamentos do que se conhece sobre determinada área, desenvolvimento de protótipos de análises de pesquisas, avaliação da situação da produção do conhecimento da área focalizada (BRANDÃO, 1986). Para Messina (1998, p. 1):

\begin{abstract}
um Estado da Arte é um mapa que nos permite continuar caminhando; um Estado da Arte é também uma possibilidade de perceber discursos que em um primeiro exame se apresentam como descontínuos ou contraditórios. Em um estado da arte está presente a possibilidade de contribuir com a teoria e prática de uma área do conhecimento.
\end{abstract}

Portanto, a realização de uma pesquisa desse tipo, possibilita a organização, a análise e a revisão de artigos de certo tema escolhido, já publicado, o que equivale à releitura, permitindo assim uma percepção do que vem sendo produzido na área e suas respectivas produções científicas. Essa escolha metodológica nos possibilitou realizar a releitura dos artigos científicos selecionados, com intuito de analisar seus objetivos. Cabe ressaltar que para análise dos dados, utilizamos a Análise de Conteúdo (BARDIN, 2010).

Conforme Bardin (2010) a Análise do Conteúdo é um processo de tipo estruturalista, que comporta duas etapas: (1) o inventário - isolar os elementos; e (2) a classificação - repartir os elementos e procurar ou impor certa organização às mensagens. Baseados nessa metodologia de organização das análises, procedemos da seguinte forma:

- $1^{\mathrm{o}} \mathrm{o}$ inventário (isolar os elementos): foram classificados os artigos da revista, os quais continham no título o descritor "saúde." Foram encontrados 39 artigos, relativos a publicações do período de 2008 a 2015.

- $2^{\circ}$ classificação (coleta de dados): a coleta de dados também foi realizada mediante o uso de algumas técnicas conforme o método de Markoni e Lakakos (2002). Com uso de tabelas, para melhor entendimento ao leitor e organização do material estudado e analisado, sendo classificados de acordo com o volume, 
número, ano, títulos e objetivos. Nessa tabela foi descrito quais objetivos foram empregadas em cada artigo.

A organização das análises dos dados foi realizada de acordo com os procedimentos indicados anteriormente, também foi feita a revisão da literatura para embasamento da pesquisa, através da consulta de diversos artigos. Em seguida, realizouse a categorização dos objetivos apresentados nos artigos do periódico, segundo a proposta de Bardin (2010), a qual envolveu: a) a identificação dos temas e sua posterior divisão em unidades de respostas; b) o recorte dos textos de acordo com os conteúdos apresentados; e c) o agrupamento e a categorização das unidades, que representam o conjunto de ideias comuns ao grupo pesquisado, apresentado nos resultados e discussões a seguir.

\section{RESULTADOS E DISCUSSÃO}

Após a realização do processo de busca e análise dos trabalhos publicados na Revista Eletrônica Ensino, Saúde e Ambiente, foram identificados 39 artigos (19,1\%), de um total de 204 publicados pela revista no período, que continham o descritor "saúde" em seus títulos.

Nos 39 artigos identificados, foi verificada uma grande variedade de trabalhos, voltados desde o comportamento alimentar, como também, produções relacionadas a pressupostos epistemológicos; formas de prevenção de doenças; relações de gênero; especificidade de estudos no campo das políticas de saúde; análises sobre o tema saúde nos livros didáticos e textos; estudos históricos sociais sobre as doenças e até produções voltadas a perspectiva sociológica nos estudos de avaliação em saúde.

Ao analisarmos as produções encontradas, pela Análise de Conteúdo (BARDIN, 2010), identificamos e categorizamos as unidades que representaram o conjunto de ideias comuns em relação aos objetivos pesquisados.

\section{Categorização dos locais do foco das investigações analisadas}

Identificamos os principais locais do foco dos estudos encontrados, onde os espaços escolares, categorização "escola", tiveram predominância, conforme pode ser observado na Tabela 1 , com percentual de 51\%. Isso significou que os espaços escolares foram os escolhidos para as atividades de investigações, abrindo um leque de ensino e 
aprendizagem em Educação em Saúde e Educação em Ciências, nos remetendo a necessidade de uma busca constante de reflexões sobre o tema nas escolas e ações voltadas a saúde individual e coletiva da sociedade em que, subentende-se aqui, que os trabalhos efetuados levaram informações para dentro da escola e lá disseminaram o tema em questão, para diferentes sujeitos envolvido. E, com base em diversos estudos, atribui-se o papel de Educação em Saúde ao ambiente escolar (BRASIL, 1997; DINIZ; OLIVEIRA; SCHALL, 2010; SCHMITZ et al, 2008).

Guimarães et al (2005) acredita que a escola é um espaço significativo na formação de crianças em processo de construção do conhecimento, o ambiente escolar pode ser considerado adequado para se trabalhar a formação de valores e hábitos favoráveis à saúde.

Tabelas 1 - Locais do foco das investigações analisadas observados nos 39 artigos analisados

\begin{tabular}{c|c|c}
\hline Locais de Investigação & Número de Artigos & Percentual \\
\hline Escola & 20 & $51,0 \%$ \\
\hline Programas e textos; livros didáticos & 11 & $28,5 \%$ \\
\hline Unidade de Saúde & 8 & $20,5 \%$ \\
\hline Total & $\mathbf{3 9}$ & $\mathbf{1 0 0 \%}$ \\
\hline
\end{tabular}

Fonte: Produção dos autores a partir da Análise de Conteúdo dos dados obtidos

Com percentual de 28,5\%, (Tabela 1), ficaram "programas e textos; livros didáticos". Os artigos realizaram discussões e análises do tema saúde na perspectiva do conceito. Além disso, também foi constatadas divulgação e aplicação de programas de saúde, visando à promoção da saúde. Constatamos que os programas de saúde, nem sempre chegam a quem precisa devido a pouca abrangência de referência e muitas vezes tropeçam na descontinuidade dos projetos.

Em relação aos temas abordados aos livros didáticos, são poucos sugestivos, em relação ao tema transversal, saúde, na sua maioria são sucintos no seu conteúdo, isso dificulta a análise do pesquisador, pois possui poucas informações. Como argumenta Soares (1996, p. 55), “o livro didático surge com a própria escola, estando presente ao longo da história, em todas as sociedades e em todos os tempos, não podendo ser compreendido isoladamente, sem que se leve em conta o contexto escolar e social". Também, conforme conclusões de Freitas e Martins (2008) as concepções de saúde encontrada nos livros didáticos, que analisaram, podem ser consideradas limitadas, porém, também proporcionam possibilidades para o professor criar e recriar, adotando mecanismos de pesquisas para problematizar de modo contextualizado as questões de saúde. 
Alves (1987, p. 50) destaca:

o fato do LD não buscar formas simples para levar o aluno a compreender e relacionar conteúdos que possibilitassem uma apropriação do conhecimento sobre seu corpo, mas, ao invés disso, optar por apresentar listas intermináveis de nomes para serem decorados.

Apesar dessas conclusões dos autores que analisaram o tema "saúde", ainda assim, o livro didático está presente nos espaços escolares e é uma das ferramentas principais utilizada pelos professores por ser de fácil acesso e de livre escolha (professores de Ciências se reúnem e escolhem o livro que irão usar na escola), porém o tema em questão é carente e cabe ao professor proporcionar ao aluno uma aprendizagem significativa, criar novas metodologias e ser capaz de perceber as limitações que o livro didático traz.

Com 20,5\%, (Tabela 1), encontramos as investigações realizadas nas "unidades de saúde". Evidenciamos que em sua maioria envolveram os profissionais da saúde; as concepções e análise dos agentes comunitários de saúde, entre outros profissionais; envolvendo também ações educativas a comunidade. O ambiente das unidades de saúde proporciona atendimento de forma próxima e acessível ao cidadão, além de ser um local para praticar boas ações educacionais e de valia na promoção da saúde, porém atendendo à necessidade de certo grupo de pacientes dos bairros da cidade, de forma restrita.

Também os profissionais das unidades de saúde e o PSE apontam, especificamente, as equipes da Estratégia de Saúde da Família (ESF) para constituir, junto com a Educação Básica e juntamente com a participação da comunidade escolar métodos para a integração e a articulação permanente entre as políticas e ações de educação e de saúde (BRASIL, 2009). Juntas realizam um trabalho de integração de importante articulação com as escolas, estas ocorrem, quando os professores estão trabalhando os conteúdos conceituais e sentem a necessidade de um especialista na área (médico, dentista ou enfermeira), que possa planejar em conjunto determinada atividade, sempre com a coordenação e supervisão de um professor. Atitudes e ideias dessa natureza traz a união entre unidades de saúde e escolas, para o bem servir da comunidade e contextualização do conteúdo com a realidade do aluno, para que essa relação entre estes profissionais possa enriquecer a Educação em Saúde no Ensino de Ciências. 


\section{Ensino, Saúde e Ambiente - V9 (3), pp. 84-99, Dez. 2016}

\section{Categorização dos objetivos empregados nos artigos}

Em relação aos objetivos dos artigos, verificamos a contribuição da "avaliação de estratégias de ensino e aprendizagem na Educação em Saúde”, com percentuais de 23\% (tabela 2), ou seja, foi nos espaços escolares que a Educação em Saúde e a formação de profissionais para o Ensino de Ciências e da área da saúde, desenvolveram formas de intervenções educativas para os sujeitos como um todo, contribuindo para o nível de bem-estar, capaz de provocar mudanças comportamentais, com intuito de influenciar o bem estar da sociedade. Venturi (2013) relata a importância das estratégias de ensino da Educação em Saúde que envolve as relações entre diferentes profissionais, para ele:

é necessário que professores e profissionais da saúde desenvolvam um trabalho em conjunto, [...] mas com planejamento e buscando os objetivos necessários para a escola e para o EC [Ensino de Ciências]: ensinoaprendizagem e construção de conhecimentos visando desenvolvimento de raciocínio, reflexão e capacidade de análise e crítica (VENTURI, 2013, p. 134).

Visualizamos que trazer formas articuladas de se trabalhar a Educação em Saúde na escola é uma estratégia que apresenta possibilidades e contribuições para a aprendizagem dos sujeitos envolvidos.

Tabela 2 - Análise das produções a partir da categorização dos objetivos observados nos 39 artigos analisados

\begin{tabular}{c|c|c}
\hline Categorias a partir dos objetivos propostos & $\begin{array}{c}\text { Número } \\
\text { de artigos }\end{array}$ & Percentual \\
\hline Avaliação de estratégias de ensino e aprendizagem na Educação em Saúde & 9 & $23,0 \%$ \\
\hline Definição e conceituação epistemológica e de percepções de saúde & 5 & $12,5 \%$ \\
\hline Difusão de informações e prevenção de doenças & 5 & $12,5 \%$ \\
\hline Avaliação do tema saúde no currículo e na abordagem escolar e em cursos & 5 & $12,5 \%$ \\
superiores & & \\
\hline Avaliação da saúde, qualidade de vida e avaliação de hábitos alimentares & 4 & $10,5 \%$ \\
\hline Contribuir/Analisar o tema saúde em livros de Ciências & 4 & $10,5 \%$ \\
\hline Avaliação do tema saúde em meios de comunicação não científicos & 3 & $8,5 \%$ \\
\hline Avaliação do papel do estágio em unidade de saúde & 2 & $5,0 \%$ \\
\hline Avaliação de temas da saúde em periódicos & 1 & $2,5 \%$ \\
\hline Avaliação motivacional de abandono de tratamentos & 1 & $2,5 \%$ \\
\hline Total & $\mathbf{3 9}$ & $\mathbf{1 0 0 \%}$ \\
\hline
\end{tabular}

Fonte: Produção dos autores a partir da Análise de Conteúdo dos dados obtidos

Em relação à categorização dos objetivos, “definição e conceituação epistemológica e de percepções de saúde"; “difusão de informações e prevenção de doenças" e "avaliação do tema saúde no currículo e na abordagem escolar e em cursos superiores", apresentaram percentuais de 12,5\%. Esses objetivos representaram o importante papel, de informar e sensibilizar a população, num todo, acerca das doenças 
transmissíveis e doenças das mais típicas e variadas, para que se adquirem hábitos saudáveis e qualidade de vida.

Em relação ao objetivo "definição e conceituação epistemológica e de percepções de saúde" podemos ver com Marinho e Silva (2015, p. 362) que "na aprendizagem da Educação em Saúde muitos saberes são construídos pelos alunos em sua vivência anterior ao seu contato com determinado conteúdo científico na escola". Nesse sentido, e tomando consciência das percepções de saúde que os alunos adquiriram no seu cotidiano, podem servir de ponto de partida para o educador articular o conhecimento prévio dos estudantes com os conhecimentos científicos.

O objetivo "difusão de informações e prevenção de doenças" nos possibilita visualizar que a criação de projetos, formação de multiplicadores e pesquisas é mais um mecanismo usado pelas autoridades da área de saúde para chamar a atenção da população quanto à necessidade de prevenção de doenças e a difusão de informações importantes, sobretudo nas comunidades mais carentes. Estes trabalhos podem envolver todos os seguimentos da sociedade, atingindo o público alvo.

Quanto ao objetivo "avaliação do tema saúde no currículo e na abordagem escolar e em cursos superiores" podemos analisar o resultado do estudo de Marinho, Silva e Ferreira (2015, p. 441) que concluem ao analisar o PCN do tema transversal saúde o "quão difícil é o entendimento da ideia de transversalidade presente em tal documento, visto que se apresenta pouco elucidativo no que concerne ao entendimento dos temas transversais". Visualizamos que um documento curricular de 1997 apresenta ideias confusas de se trabalhar com a questão da saúde na escola, sendo pertinente sua discussão e problematização. Tais autores também apresentam uma dificuldade que os professores, em sua maioria, encontram em trabalhar e discorrer essa temática, pois acabam não reconhecendo a saúde como conteúdo de ensino.

Os objetivos, "contribuir/analisar o tema saúde em livros de Ciências" e “avaliação da saúde, qualidade de vida e avaliação de hábitos alimentares", apresentaram percentuais de $10,5 \%$. Em relação ao estudo do tema saúde em livros didáticos podemos verificar que, segundo as autoras Freitas e Martins (2008), no que diz respeito aos conteúdos de saúde nos livros didáticos, concluíram que, os autores dos livros didáticos, priorizam frequentemente os conteúdos de aspectos anatômicos e fisiológicos e questões atuais acabam não sendo contempladas nas obras. Assim, apontam a escassez de análises que envolvem o tema da saúde nos livros didáticos de Ciências. Já sobre a "avaliação da saúde, qualidade de vida e avaliação de hábitos 


\section{Ensino, Saúde e Ambiente - V9 (3), pp. 84-99, Dez. 2016}

alimentares" podemos perceber que no ambiente escolar, seria pertinente que os educadores pudessem utilizar várias estratégias de ensino, contribuindo para a melhoria na alimentação dos sujeitos (DAVANÇO et al., 2004; BIZZO e LEDER, 2005).

O objetivo "avaliação do tema saúde em meios de comunicação não científicos" com percentual de $8,5 \%$ objetivou a avaliar o tema saúde em locais da mídia, efetivando assim a avaliação de compartilhar significados mediante o intercâmbio de informação. Bueno (2009, p. 104), discorre que “a comunicação moderna deve ser personalizada, não pode partir do pressuposto de que um mesmo assunto interessa a todo mundo, porque há visões de mundo e experiências diferentes de um público para outro". É neste cenário globalizado que os canais de comunicação garantem intervenções, mantendo o vínculo entre Ciência e mídia. Alguns estudos sobre reportagens jornalísticas podem ser um recurso didático em sala de aula, também mostram que estas reportagens apresentam conteúdos científicos de forma correta, corroborando com a extensão do tema saúde, trazendo informações a população.

“Avaliação do papel do estágio em unidade de saúde" foi um objetivo que obteve um percentual de 5\%. Nesse notou-se a avaliação dos estágios dos alunos (Curso de Enfermagem), e consequentemente as análises das experiências e vivências dos autores, nos seus estágios, os quais contribuíram para sua profissionalização, pois é nesse momento que eles percebem o quanto a Educação em Saúde carece de ações, que tangem a promoção da saúde e que essas ações, podem gerar mudanças positivas e transformadoras na sociedade.

Por fim a "avaliação de temas da saúde em periódicos" e "avaliação motivacional de abandono de tratamentos," apresentaram percentuais de 2,5\%. Em relação à avaliação do tema em periódicos observamos que Venturi e Mohr (2011) apresentam um estudo em que constataram que as publicações abordando os temas de Educação em Saúde nos periódicos de Educação em Ciências ainda são escassas. Ainda assim, tendo em vista a relevância do tema saúde, na maioria dos periódicos é tratada principalmente sob a ótica diagnóstica, ou seja, o levantamento de dados e as possíveis associações com diferentes patologias. Constataram também que as concepções sobre o tema foram avaliadas, assim como as formas de procedimentos, suas aplicações e suas implicações nas pesquisas. Já os trabalhos relacionados ao abandono dos pacientes que estavam em tratamento, visa promover projetos de motivação aos pacientes que já estão fragilizadas pelas doenças é ações que vão de encontro com o conceito de saúde. 
Devido à baixa estima dos pacientes adoentados, passou-se a dar importância aos programas que privilegiassem ações educativas relacionadas a informar os pacientes e às mudanças de atitude dos pacientes. Fraga (2005, p. 28) destaca o quanto a noção de estilo de vida ativo "dissemina-se como um dos mais preciosos elementos de promoção da saúde e bem estar, qualidade de vida e longevidade". Destacar que informar é importante, mas não suficiente para gerar as mudanças necessárias que promovam a prevenção da doença e a não desistência desses pacientes.

No que tange aos objetivos e suas categorizações, na Tabela 2, na maioria dos artigos, os autores, avaliaram, analisaram o tema saúde, nas mais diversas áreas ou lugares, como no âmbito escolar, unidade de saúde, espaços acadêmicos, programas, textos, nos livros didáticos, mas na sua maioria foram em espaços escolares que se destacaram, ganharam voz, buscando as mais variadas estratégias de ensino e aprendizagem contribuindo com o Ensino de Ciências e a Educação para a Saúde.

Vale ressaltar que os objetivos aqui considerados, se limitaram a tão somente análise, conforme lidos nos artigos e não foram estudados se os objetivos dos artigos foram alcançados.

\section{CONSIDERAÇÕES FINAIS}

O estudo levantou um número de 39 publicações científicas $(19,1 \%)$, de um total de 204 publicados pela revista no período, que apresentaram o tema "saúde", no entanto, ainda bastante centradas no âmbito escolar, no que diz respeito à atenção básica e a perspectiva da Saúde Coletiva. Além disso, também foi concluído que nos espaços escolares se desenvolveram atividades de investigações, de ensino-aprendizagem da Educação em Saúde e Educação em Ciências, assim como, reflexões e problematizações, isso, significou uma forma de abordar o tema saúde dando significado ao assunto nas escolas.

Como resultados das análises dos objetivos analisados evidenciamos as estratégias de ensino e a avaliação do tema saúde, como predominante, as quais os autores dos artigos avaliaram, analisaram, conceituaram e desenvolveram diversas estratégias de ensino. Nesse contexto, podemos evidenciar que as publicações científicas da revista eletrônica "Ensino, Saúde e Ambiente", está a contribuir para o resultado da disseminação de conhecimentos relacionados à Educação em Saúde e ao Ensino de Ciências. 
Ensino, Saúde e Ambiente - V9 (3), pp. 84-99, Dez. 2016

\section{REFERÊNCIAS}

ALVES, N. C. A saúde na sala de aula: uma análise nos livros didáticos. Cadernos CEDES, v. 18, p. 38-53, 1987.

BARDIN, L. Análise de conteúdo. Lisboa: Edições 70, 2010.

BIZZO, M. L. G.; LEDER L. Educação nutricional nos parâmetros curriculares nacionais para ensino fundamental. Ver. Nutr., v. 18, p. 661-667, 2005.

BRANDÃO, Z.; BAETA, A. M. B.; ROCHA, A. D. C. Evasão e repetência no Brasil: a escola em questão. 2 ed. Rio de Janeiro: Dois Pontos, 1986.

BRASIL. Secretaria de Educação Fundamental. Parâmetros curriculares nacionais: apresentação dos temas transversais, ética. Brasília: MEC/SEF, 1997.

Presidência da República. Decreto no 6.286, de 5 de dezembro de 2007.

Institui o Programa Saúde na Escola - PSE, e dá outras providências. Diário Oficial da União, Poder Executivo, Brasília, DF, 2007.

Ministério da Saúde; Ministério da Educação. Programa Saúde na Escola.

Brasília: Editora do Ministério da Saúde, 2008a.

Ministério da Saúde. Secretaria-Executiva. Mais saúde: direito de todos: 20082011. Brasília: Editora do Ministério da Saúde, 2008b.

Ministério da Saúde. Secretaria de Atenção à Saúde. Departamento de Atenção Básica. Saúde na escola / Ministério da Saúde, Secretaria de Atenção à Saúde, Departamento de Atenção Básica. Brasília: Ministério da Saúde, 2009.

BUENO, C. W. Comunicação Empresarial: Políticas e Estratégicas. São Paulo: Saraiva, 2009.

DAVANÇO, G. M.; TADDEI, J. A. A. C.; GAGLIANONE, C. P. Conhecimentos, atitudes e práticas de professores de ciclo básico expostos e não expostos a curso de educação nutricional. Rev. Nutr., v. 17, p. 177-84, 2004.

DINIZ, M. C. P.; OLIVEIRA, T. C.; SCHALL, V. T. Saúde como compreensão de vida: avaliação para inovação na educação em saúde para o ensino fundamental.

Revista Ensaio: Pesquisa em Educação em Ciências, Belo Horizonte, v. 12, n. 1, p.119-144, jan./abr. 2010.

FONSECA, J. P. Aluno, paciente, cidadão: a saúde escolar em questão. CONCEIÇÃO, José Augusto Nigro (Org.). Saúde escolar: a criança, a vida e a escola. São Paulo: Sarvier, 1994. p. 23-32.

FRAGA, A. B. Exercício da informação: governo dos corpos no mercado da vida ativa. Porto Alegre: UFRGS, 2005. 175 f. Tese (Doutorado em Educação) - Programa de Pós - Graduação em Educação, Faculdade de Educação, Universidade Federal do Rio Grande do Sul, Porto Alegre, 2005. 
FREITAS, E. O.; MARTINS, I. Concepções de saúde no livro didático de ciências. Ensaio: Pesquisa em Educação em Ciências, Belo Horizonte, v. 10, n. 2, p. 222-248, jul./dez. 2008.

GUIMARÃES, T. A. A. et al. A concepção de professores de ensino fundamental do município de Jequié, BA sobre saúde-doença. Rev. Saúde. Com, v. 1, n. 2, p. 95-99, 2005

LEVY, S. N.; SILVA, J. J. C. D.; CARDOSO, I. F. R.; WERBERICH, P. M.;

MOREIRA, L. L.; MONTIANI, H.; CARNEIRO, R. M. Educação em saúde: histórico, conceitos e propostas. In: Educação em saúde: histórico, conceitos e propostas. Brasil: Ministério da Saúde, 1997.

MARCONI, M. A.; LAKATOS, E. M. Técnicas de pesquisa: planejamento e execução de pesquisas, amostragens e técnicas de pesquisa, elaboração, análise e interpretação de dados. 5 ed. São Paulo: Atlas, 2002.

MARINHO, J. C. B.; SILVA, J. A. Concepções e implicações da aprendizagem no campo da educação em saúde. Revista Ensaio: Pesquisa em Educação em Ciências, Belo Horizonte, v.17, n. 2, p. 351-371, 2015.

MARINHO, J. C. B.; SILVA, J. A.; FERREIRA, M. A educação em saúde como proposta transversal: analisando os Parâmetros Curriculares Nacionais e algumas concepções docentes. História, Ciências, Saúde - Manguinhos, Rio de Janeiro, v. 22, n. 2, p. 429-443, abr./jun. 2015.

MESSINA, G. Estudio sobre el estado da arte de la investigacion acerca de la formación docente en los noventa. Organización de Estados IberoAmericanos para La Educación, La Ciência y La Cultura. In: Reúnion de consulta técnica sobre investigación en formácion del professorado. México,1998.

MINISTÉRIO DA SAÚDE. Diretrizes da Educação para a Saúde. Educação em Saúde nas Unidades Federadas. Brasília: Ministério da Saúde, 1980.

MINISTÉRIO DA SAÚDE. Geras - Documento Básico. Brasília: MS/Geras, 1993.

MOHR, A. A natureza da Educação em Saúde no ensino fundamental e os professores de ciências. Santa Catarina. 2002. Tese (Doutorado). Programa de PósGraduação em Educação, Centro de Ciências da Educação - Universidade Federal de Santa Catarina, 2002.

REVISTA ELETRÔNICA ENSINO, SAÚDE E AMBIENTE. Foco e escopo. Disponível em: <http://ensinosaudeambiente.uff.br/index.php/ensinosaudeambiente/ about/editorialPolicies\#focusAndScope>. Acesso em: 12 de nov. 2015.

SANTOS, A. R. Metodologia científica: a construção do conhecimento. Rio de Janeiro: DP \& A, 1999.

SANTOS, R. N.; ALCÂNTARA-ELIEL, R.; ELIEL, O. A ciência e o novo estado do conhecimento: a contribuição da ciência da informação. Enc. Bibli: R. letr. Bibliocon. Ci. Inf., n. 22, p. 16-29, 2006. 
SCHALL, V. T.; STUCHINER, M. Educação em saúde: novas perspectivas. Cad. Saúde Pública, v. 2, p. 4-5, 1999

SCHMITZ, B. A. S. et al. A escola promovendo hábitos alimentares saudáveis: uma proposta metodológica de capacitação para educadores e donos de cantina escolar. Cad. Saúde Pública, Rio de Janeiro, v. 24, sup. 2, p. 312-322, 2008.

SILVA, P. M. M.; JULIANI, C. M. C. M. Educação em Saúde. Universidade do Estado de São Paulo (UNESP). São Paulo, 2010. Disponível em: < http://www. datasus. gov.br/temas/educacaosaude/educacaosaude,htm> . Acesso em: 20 abr. 2016.

SOARES, M. B. Alfabetização no Brasil: o estado do conhecimento. Brasília: INEP/Santiago: Reduc, 1989.

SOARES, M. B. Um olhar sobre o livro didático. Presença Pedagógica, v. 2, n. 12 , nov./dez. 1996.

VASCONCELOS, E. M. Educação popular como instrumento de reorientação das estratégias de controle das doenças infecciosas e parasitárias. Cadernos de Saúde Pública, v. 14, p. 39-57, 1998.

VENTURI, Tiago. Educação em Saúde: investigando relações entre Professores e Profissionais da Saúde. Dissertação (Mestrado). Programa de Pós-Graduação em Educação Científica e Tecnológica, Universidade Federal de Santa Catarina - UFSC. Florianópolis, 2013.

VENTURI, T.; MOHR, A. Análise da Educação em Saúde em publicações da área da Educação em Ciências. In: Atas do VIII Encontro de Pesquisa em Educação e I Congresso Iberoamericano de Investigação e Ensino de Ciências, UNICAMP, SP: 2011.

WEINECK, J. Atividade física e esporte: para quê? Barueri: Manole, 2003. 